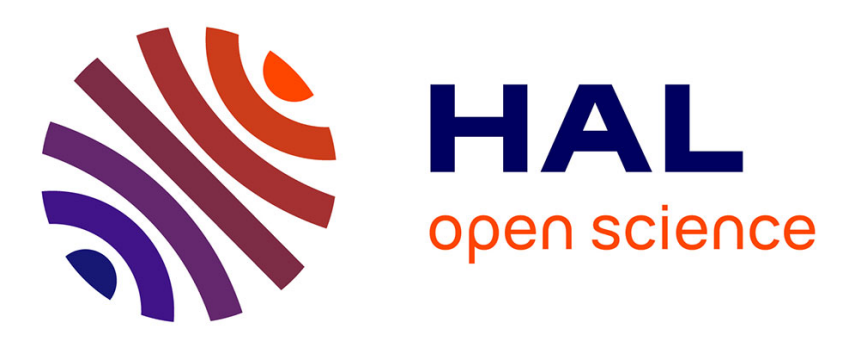

\title{
Modeling of the dynamic behavior of systems with rolling elements
}

K. de Moerlooze, F. Al-Bender, H. van Brussel

\section{To cite this version:}

K. de Moerlooze, F. Al-Bender, H. van Brussel. Modeling of the dynamic behavior of systems with rolling elements. International Journal of Non-Linear Mechanics, 2010, 46 (1), pp.222. 10.1016/j.ijnonlinmec.2010.09.003 . hal-00701878

\section{HAL Id: hal-00701878 \\ https://hal.science/hal-00701878}

Submitted on 27 May 2012

HAL is a multi-disciplinary open access archive for the deposit and dissemination of scientific research documents, whether they are published or not. The documents may come from teaching and research institutions in France or abroad, or from public or private research centers.
L'archive ouverte pluridisciplinaire HAL, est destinée au dépôt et à la diffusion de documents scientifiques de niveau recherche, publiés ou non, émanant des établissements d'enseignement et de recherche français ou étrangers, des laboratoires publics ou privés. 


\section{Author's Accepted Manuscript}

.

NON-LINEAR

MECHANICS

Modeling of the dynamic behavior of systems with rolling elements

K. De Moerlooze, F. Al-Bender, H. Van Brussel

PII:

S0020-7462(10)00135-6

DOI:

doi:10.1016/j.ijnonlinmec.2010.09.003

Reference:

NLM 1758

To appear in: International Journal of NonLinear Mechanics

Received date: 9 March 2009

Revised date: 3 September 2010

Accepted date: 6 September 2010

Cite this article as: K. De Moerlooze, F. Al-Bender and H. Van Brussel, Modeling of the dynamic behavior of systems with rolling elements, International Journal of Non-Linear Mechanics, doi:10.1016/j.ijnonlinmec.2010.09.003

This is a PDF file of an unedited manuscript that has been accepted for publication. As a service to our customers we are providing this early version of the manuscript. The manuscript will undergo copyediting, typesetting, and review of the resulting galley proof before it is published in its final citable form. Please note that during the production process errors may be discovered which could affect the content, and all legal disclaimers that apply to the journal pertain. 


\title{
Modeling of the dynamic behavior of systems with rolling elements
}

\author{
K. De Moerlooze ${ }^{\mathrm{a}, *}$, F. Al-Bender ${ }^{\mathrm{a}}$, H. Van Brussel $^{\mathrm{a}}$ \\ ${ }^{a}$ Katholieke Universiteit Leuven, Mechanical Engineering Department, Division PMA, \\ Celestijnenlaan 300B, B-3001 Heverlee (Belgium)
}

\begin{abstract}
Bearings, friction wheels, cams etc. are widely employed elements in machine construction. The modeling of the dynamics of rolling friction plays therefore a crucial role in the simulation and optimization of such systems. This paper describes a recently developed transient-rolling-friction model with its application to different generic dynamical systems, to simulate the effects of rolling friction or traction on the dynamics of mechanical systems. The results, which are expressed in terms of the complex stiffness arising in the rolling-contact patches, show that the behavior depends on both the amplitude and the frequency of oscillation. For low amplitudes and frequencies, the behavior is quasi linear. For increasing amplitudes and frequencies, however, strong nonlinearities appear, leading to complex behavior.
\end{abstract}

Key words: contact mechanics, Hertzian contact, longitudinal creepage, lateral creepage, spin, tractive rolling dynamics, dry friction

\section{Introduction}

Rolling elements are widely used in machine construction for motion guidance and transmission, in various applications, including robotics and positioning systems, railway and vehicle applications. The growing demands for accu-

\footnotetext{
*corresponding author: Tel.: +32-16-32-24-81; fax: +32-16- 32-29-87.

Email addresses: Kris.DeMoerlooze@mech.kuleuven.be (K. De Moerlooze), Farid.Al-Bender@mech.kuleuven.be (F. Al-Bender), Hendrik.VanBrussel@mech.kuleuven. be (H. Van Brussel)
} 
racy, performance, and reliability in machines require an accurate modeling of friction in their moving parts.

Positioning and machining devices often employ rolling elements, such as ball bearings and plain bearings (see, e.g. $[1,2,3,4]$ ). The study of rolling-element bearings was and still is an important research topic $[5,6,7,8,9,10,11]$. These elements introduce nonlinear frictional behavior, which often mortgages accurate and fast positioning. Furthermore, the replacement of failed or damaged elements constitutes a considerable part of the maintenance costs in industrial applications and maintenance schemes.

The study of traction in tyre-road contacts $[12,13]$ can lead to significant improvements in the drive comfort, handling and reliability of vehicles. Similar research is performed and is still ongoing in the field of railway applications $[14,15,16,17,18]$, where noise and vibrations such as squeaking and rattling while braking and taking bends, and associated wear, are of great concern $[19,20,21]$.

The preceding examples point to the exigency of simulating and optimizing rolling contacts. A profound study of the tractive forces in the contact between a rolling element and its counter surface is therefore a pertinent issue, together with the investigation of the reciprocal influence of friction and structural dynamics.

The traction stress field in the contact of a body of revolution and its mating surface, originates from the relative motion between points on the contacting bodies. When an elastic body of revolution rolls with traction over another, the traction field in the contact patch changes progressively with the traversed distance and the prevailing creepages. This traction field, which is generally dynamic, determines the traction forces developed in the system. During the past decades, there has been a significant amount of research into rolling elements, which remained, however, restricted to steady-state rolling. Consequently, nowadays, the steady-state gross rolling process is well understood, while transient effects still need a profound investigation. 
The transition period, building up to steady, gross rolling, which is denominated the pre-rolling period, is characterized by rate independent hysteresis behavior with nonlocal-memory effect [22]. In an earlier publication [23], the authors present a transient model which is able to simulate the motion and traction stresses in a tractive rolling contact, subjected to a constant creepage. This model has been validated in Ref. [24] for the specific case of a ball rolling in a $\mathrm{V}$-grooved track. In the present paper the application of the model is extended towards dynamical systems, in which the creepage is no longer constant over time, but depends on the dynamics of the constituent system.

In applications where transient conditions are expected, such as vibrations, accelerations or decelerations, the dynamics of the system play a part in the analysis. Gupta proposes an advanced approach to simulate the rolling element motion in a general way for both roller and ball bearings [25], based on a time integration of the general differential equations of motion, and a calculation of the external forces acting on the rolling element. The determination of the external forces relies on the applied bearing load, the roller-race and the roller-cage interactions, together with the lubrication effects. In his analysis, the traction forces are based on steady-state elastohydrodynamic lubrication theory. Abascal [26] studies the transient dynamic friction in two-dimensional contacts, using the boundary element method. Spiegelberg et al. [27] study the transient friction effects in the contact between a cylinder and two flat plates, using a brush model. Overall, the knowledge of rolling friction under transient conditions is somewhat limited, while there is an obvious need for such knowledge, and for the possible models it can yield, for the purpose of characterizing the dynamics of systems comprising rolling elements.

The objective of this study is to simulate the transient rolling-friction behavior together with the dynamics of generic systems comprising rolling elements. The used rolling-friction model, based on a Winkler bedding and an appropriate choice of the flexibility parameter $L$, yields a linear approximation with a solu- 
tion close to the exact theory [23]. The transparency and the easy application of the theory is advantageous for implementing the model in larger systems, i.e. including system dynamics. The dynamics of the system will affect the developed friction behavior, while the transient effects of the friction will, in their turn, influence the system's behavior. This interaction is the underlying theme of this paper.

The paper is structured as follows. Section 2 outlines the rolling friction model, which is used in the subsequent case studies. In section 3 , the dynamics of a ball on a flat plane is investigated, where the rotation of the ball is affected by the imposed motion of the contacting flat. In section 4, a two degree-of-freedom model is presented, namely the motion of a ball in between two flat planes, where the system is excited through the motion of the upper plane. The last case study in section 5 comprises the motion of a ball in between two V-grooved tracks. In this case, the kinematic spin motion of the ball is added to the dynamical creepage in the contact. Finally, appropriate conclusions are drawn.

\section{Transient rolling of a sphere on a plane surface}

Based on $[23,24]$, this section delineates the basic theory for modeling the transient, tractive rolling of a sphere on a plane rigid surface. The rolling situation is depicted in Fig. 1. The sphere, of radius $R$, being loaded by a force normal to the plane surface, $W$, rolls in the $x$-direction such that its centre translates at a velocity $V$, while being subjected to creepages (see further).

\subsection{Problem specification}

The rolling problem is specified by determining (i) the contact patch size and pressure distribution and (ii) the rolling creepages, as follows. 


\subsubsection{Normal stresses and contact patch}

When an elastic sphere, of radius $R$ and equivalent modulus of elasticity $E_{e}$, is pressed against a rigid plane surface with a load $W$, we have:

- The contact patch will be the circular region $A=\left\{(x, y): x^{2}+y^{2} \leq a^{2}\right\}$, where the radius is given by

$$
a=\left(\frac{3 W R}{4 E_{e}}\right)^{1 / 3}
$$

- The normal stress, $p_{z}$, is given by:

$$
p_{z}(x, y)=p_{0} \sqrt{1-(x / a)^{2}-(y / a)^{2}} \text { with } p_{0}=\frac{3 W}{2 \pi a^{2}}
$$

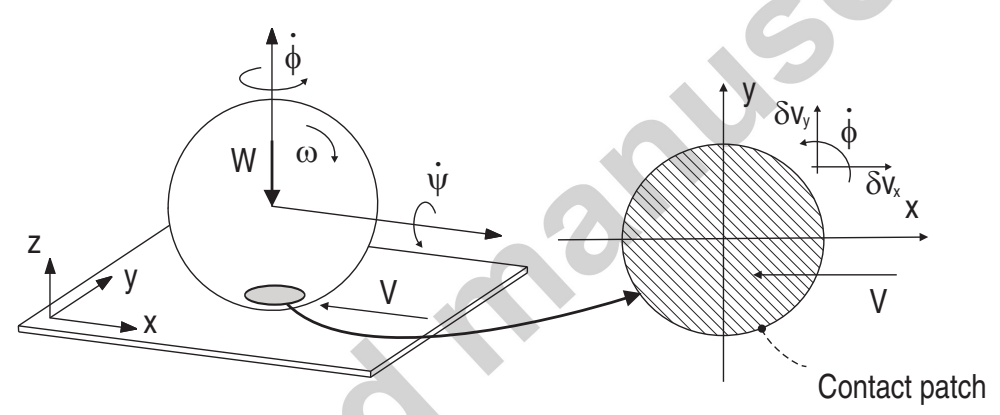

Figure 1: Overview of the rolling motion. (reproduced with permission from [23])

\subsubsection{The creepages}

Creepage, sometimes called rigid slip, is the difference between surface velocities of the rolling objects in the contact patch, when the objects are assumed

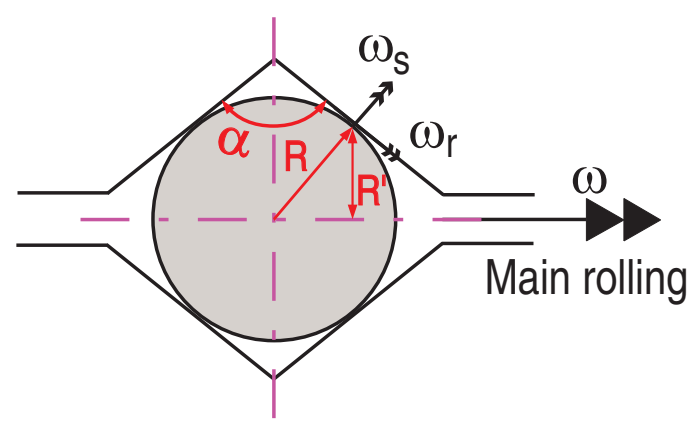

Figure 2: The configuration of V-groove rolling. 
rigid. Referring to Fig. 1, if the sphere's geometrical centre translates with a velocity $V$, while the sphere is spinning around its $x, y$ and $z$ axes with angular speeds $\dot{\psi}, \omega$ and $\dot{\phi}$ respectively, the creepages are defined by:

$$
\left\{\begin{array}{l}
\delta V_{x}=-\omega R+V \text { the longitudinal creepage } \\
\delta V_{y}=\dot{\psi} R \text { the lateral creepage } \\
\dot{\phi} \text { the spin creepage. }
\end{array}\right.
$$

Creepage can originate from (i) the kinematics of the system, or (ii) from the dynamics of the system. The case (i) can be illustrated by the rolling of a ball in a V-grooved track. Assuming that the velocity of the ball is low enough to avoid dynamic effects, or equivalently that the ball is massless, the creepage in the contact is that of pure spin, due to kinematics of the system. This is illustrated in Fig. 2. In case (ii), the creepage originates from the dynamics of the ball with respect to the track surface. Examples of this case can be found in high speed spindle bearings, in which the high accelerations applied by the electric motors result in slipping of the bearing balls in their raceways. Similar effects can occur in ball-screw nuts of dynamically driven systems.

The problem of an elastic sphere, tractively rolling on a rigid plane, can be reduced to that in which the contact patch is stationary in space and time. This is accomplished by assuming the rigid plane upon which the sphere is rolling to be moving in the opposite direction with a velocity $\mathbf{V}$ (see Fig. 1.)

\subsection{Constitutive equations}

\subsubsection{The slip equation}

In order to arrive at an equation to describe the kinematics of the surface points lying inside the contact patch $A$, one follows a point on the surface of the sphere, which enters the contact patch and mates, at the entrance, with a counterpoint on the rigid plane. Owing to the creepage, the point on the surface of the sphere will have to deform in the contact plane, by an amount $\mathbf{u}=(u, v)$ being called the displacement, which is generally function of space and time. The slip $\mathbf{s}$ is defined as the relative velocity between mating points 
in the interface. We obtain the following differential equation for the plane deformations:

$$
\mathbf{s}=\mathbf{c}-V \frac{\partial \mathbf{u}}{\partial x}+\frac{\partial \mathbf{u}}{\partial t}
$$

with $\mathbf{c}=\left(\delta V_{x}-\dot{\phi} y, \delta V_{y}+\dot{\phi} x\right)$.

Generally, $V=V(t)$. If the creepage is scaled with $V$, then one can rewrite the previous equation in terms of the traversed rolling distance, $q$ by making use of the substitution:

$$
q=\int_{0}^{t} V(\tau) d \tau
$$

Substituting this into Eq. 4 and normalizing the creepage and the spin by the rolling velocity results in:

$$
\mathbf{S}=\mathbf{C}-\frac{\partial \mathbf{u}}{\partial x}+\frac{\partial \mathbf{u}}{\partial q}
$$

where $\mathbf{S}=\mathbf{s} / V=\left(S_{x}, S_{y}\right)$ is the relative slip and $\mathbf{C}=\mathbf{c} / V=\left(\xi_{x}-\frac{\phi y}{a}, \xi_{y}+\frac{\phi x}{a}\right)$ is the relative creepage, with $\xi_{x}=\frac{\delta V_{x}}{V}, \xi_{y}=\frac{\delta V_{y}}{V}, \phi=\frac{\dot{\phi} a}{V}$.

\subsubsection{Traction-displacement relationship: A linearized theory}

Here, the relationship between surface displacement $(u, v)$ and the tangential traction field $\left(p_{x}, p_{y}\right)$ for the case of no slip, is determined using a "Winkler bedding" model. In this approximating approach, the surface of the elastic object is considered to be covered with elastic bristles in the normal direction with constant stiffness for tangential deformations, being characterized by the flexibility parameter $L$, such that the following relation holds for no-slip conditions:

$$
(u, v)=L \cdot\left(p_{x}, p_{y}\right)
$$

The flexibility parameter $L$ depends on the elasticity characteristics of the sphere and the magnitude of the creepages, and is given by:

$$
L=\frac{L_{1}\left|\xi_{x}\right|+L_{2}\left|\xi_{y}\right|+L_{3}|\phi|}{\sqrt{\xi_{x}^{2}+\xi_{y}^{2}+\phi^{2}}}
$$

The formulas for the parameters $L_{1}, L_{2}$ and $L_{3}$ are given in Appendix B. 


\subsubsection{The traction limit and slip conditions}

Equation 7 is no longer valid when slip occurs between the mating points. This will be the case when the condition

$$
\left\|\left(p_{x}, p_{y}\right)\right\| \leq \mu p_{z}
$$

is violated. Here, $p_{z}$ is given by Eq. 2 and $\mu$ is a local coefficient of friction. This condition 9 is known as the traction bound, which results in a relationship for determining the tangential tractions:

$$
\left(p_{x}, p_{y}\right)=\left\{\begin{array}{c}
\frac{(u, v)}{L} ;\left\|\left(p_{x}, p_{y}\right)\right\| \leq \mu p_{z} \\
\mu p_{z} \frac{(u, v)}{\|(u, v)\|} \text { elsewhere }
\end{array}\right.
$$

In this analysis, the local coefficient of friction is assumed to be constant in time and in contact condition. However, in real contacts, the coefficient of friction may vary owing to many factors such as variable contact pressure and/or wear caused by (partial) slip. In a more refined analysis, these effects can be taken into account. However, the error committed by neglecting them, appears to be acceptable [24], which justifies assuming a constant coefficient of friction for the time being.

\subsubsection{Initial conditions}

The final necessary ingredient for solving the rolling-contact problem is the specification of the initial traction field present in the contact prior to commencement of (pre)rolling motion. This traction field is induced by the Hertzian loading of the rolling bodies, in the normal direction, when friction is present between the contacting surfaces. The procedure for calculating this traction field is detailed in [23]. Obviously, this initial traction field will determine the transition trajectory to steady-state rolling. However, the traction field pertaining to steady-state rolling is independent of the initial traction field (see [23]). Thus, if periodic rolling involves passing through the steady-state traction field, it will, consequently, be independent of the initial conditions. In the simulation experiments presented in 


\section{the remainder of this paper, which consider only sinusoidal motion, one may therefore assume any arbitrary initial traction field, e.g. a null field, without loss of generality.}

\subsection{Numerical implementation and calculation of the traction forces and mo- ment}

In order to solve the system of equations presented above, a numerical procedure was developed in which the contact patch was discretized into a set of nodes. Starting from any given initial traction field, the new displacement distribution $(u, v)$ at every time step, is obtained by shifting the previous $u$ and $v$ vectors by the rolling increment $\Delta q$ and augmenting it with the calculated extra displacement. Since the rolling increment is generally independent of the grid size, the shifting must be performed by $2 \mathrm{D}$ bilinear interpolation of the displacement distribution. Afterwards, the traction bound is calculated and the traction limit is verified. For details, the reader is referred to [23]. When the traction fields have been determined, the resultant traction forces and traction moment are calculated by quadrature of the fields over the contact patch:

$$
\begin{gathered}
F_{x}=\int_{-a}^{a} \int_{-a(y)}^{a(y)} p_{x}(x, y) d x d y \\
F_{y}=\int_{-a}^{a} \int_{-a(y)}^{a(y)} p_{y}(x, y) d x d y \\
M_{z}=\int_{-a}^{a} \int_{-a(y)}^{a(y)} p(x, y) r(x, y) d x d y
\end{gathered}
$$

with $r(x, y)$ being the radial distance from the centre of the contact patch to the location of the node. Figure 3 shows an example of a transient simulation for the case of rolling with longitudinal creepage, where the friction force $F_{x}$ is calculated using Eq. 11. 


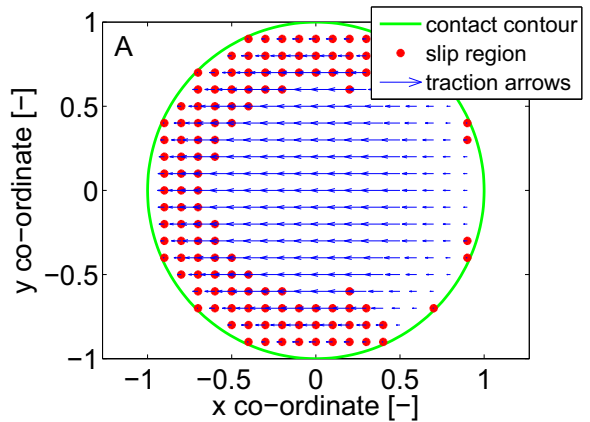

(a)

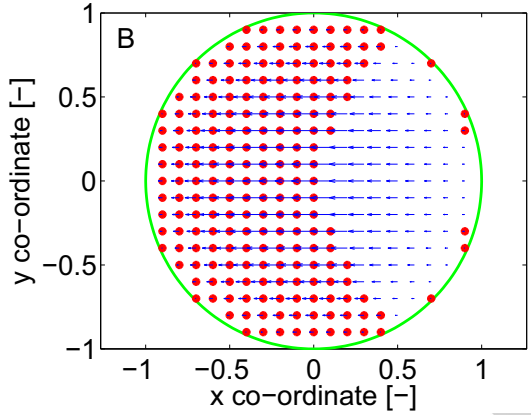

(b)

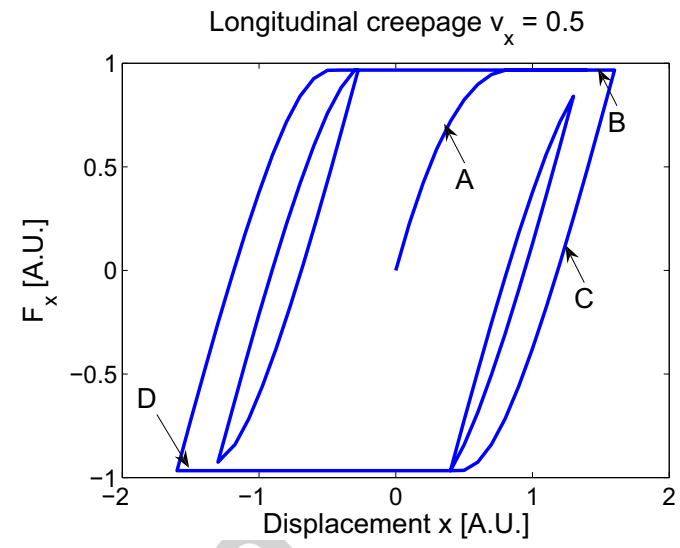

(c)

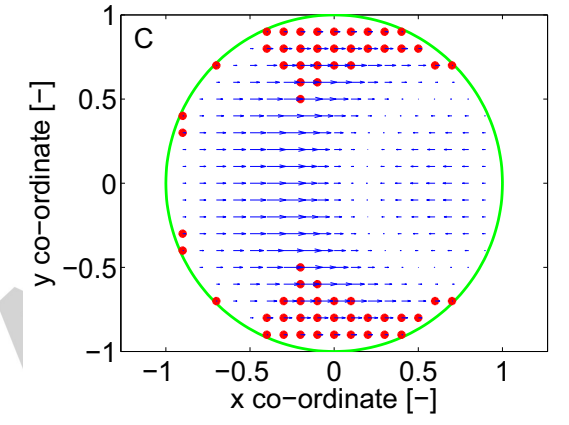

(d)

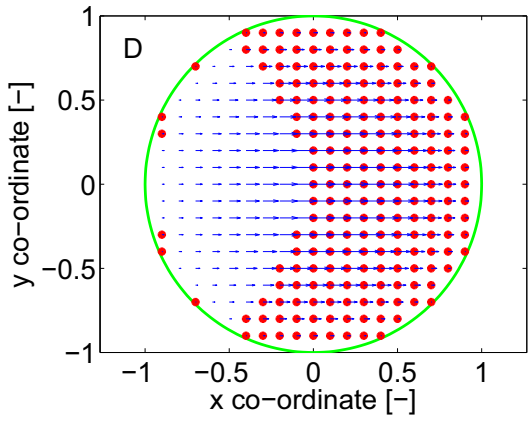

(e)

Figure 3: The results for transient (pre-)rolling with longitudinal creepage. In the middle figure, the hysteresis plot is depicted, the other figures depict the local traction field. (reproduced with permission from [23]) 


\subsection{Normalization of the model}

Before going into the case studies, and in order to facilitate analysis and presentation, we consider it useful first to normalize the problem with respect to its parameters, using dimensional analysis, so as to permit an easier analysis of the results and provide better insight into the behavior. For this purpose, the lengths are rescaled by $a$, the masses by $I / a^{2}$ ( $I$ being the moment of inertia of the sphere about an axis through its centre), and the times by $\sqrt{I /\left(p_{0} a^{3}\right)}$. The frequency of excitation is made dimensionless via:

$$
f_{t}^{*}=f_{t} \sqrt{\frac{I}{p_{0} a^{3}}} .
$$

With

$$
I=\frac{8}{15} \pi R^{5} \rho
$$

for a solid sphere, we obtain

$$
f_{t}=f_{t}^{*} \sqrt{\frac{15 E}{4 \rho} \frac{a^{2}}{\pi R^{3}}},
$$

which gives the conversion relationship between dimensional and dimensionless frequency.

In the following sections, all results are given in dimensionless form, where the $*$ is dropped for convenience.

\section{Case 1: A sphere rolling on a plane}

\subsection{System description}

As a first case to study the influence of dynamics on the frictional behavior of a system comprising a rolling element, the system depicted in Fig. 4 is treated. The rolling sphere has one degree-of-freedom, namely its (frictionless) rotation around the $z$ axis. A massless flat plate is pressed against the sphere, resulting in a circular contact patch. Obviously, the dynamic behavior of the sphere is affected by the traction between plane and sphere. A prescribed tangential displacement $x_{t}(t)$, is imposed on the upper plane, while the effect on the motion 


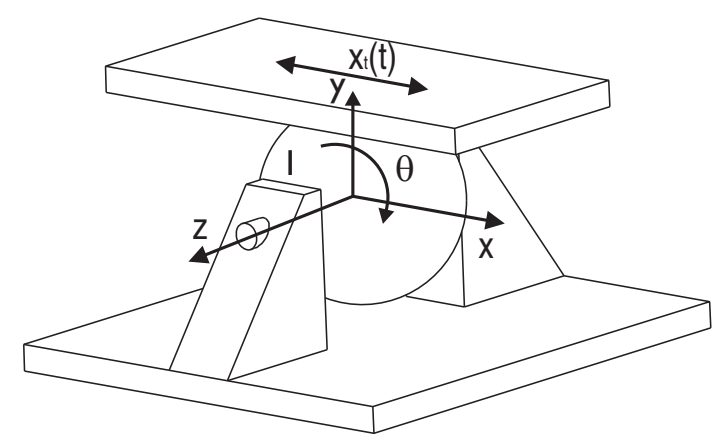

Figure 4: Rolling dynamics case 1: A 1-DOF system.

of the sphere and the friction force in the contact is determined. The rotation of the sphere is determined by the following equation:

$$
\ddot{\theta}=\mathcal{F}\left(\theta, x_{t}, \text { his }\right)
$$

where $\mathcal{F}$ is the nonlinear frictional force, which depends on the position of the upper plate, $x_{t}$, the rotation of the sphere $\theta$, and the history of the motion, his. The simulation is carried out as follows. At every time step, the friction force is calculated using the rolling friction model of section 2. For given geometrical, material and load properties of the system, the input to this model is the longitudinal creepage in the contact, given by:

$$
\xi_{x}=\frac{\dot{x_{t}}-R \dot{\theta}}{\dot{x_{t}}}
$$

The friction force acting on the sphere constitutes the input to the numerical scheme to solve Eq. 17. Since the only damping originates from the slip of the ball on the flat surface, the system will not damp out entirely. For low oscillation amplitudes, the friction force will become a linear function of the displacement, as can be observed on the hysteresis curve of Fig. 3(c), at commencement of motion reversal. In this region, very little damping will occur, and the stiffness is given by the slope of the hysteresis curve at that point. Therefore, a Newmark scheme [28] is used to solve Eq. 17, which is appropriate 


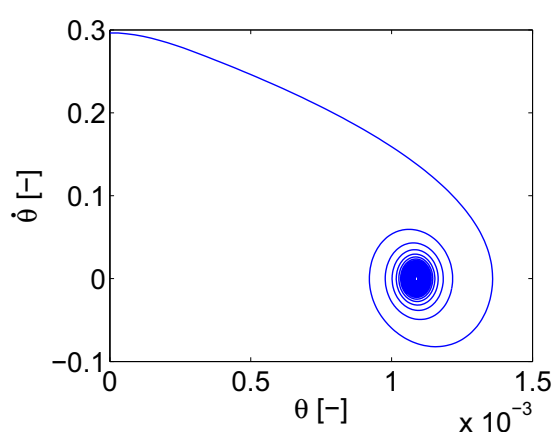

(a)

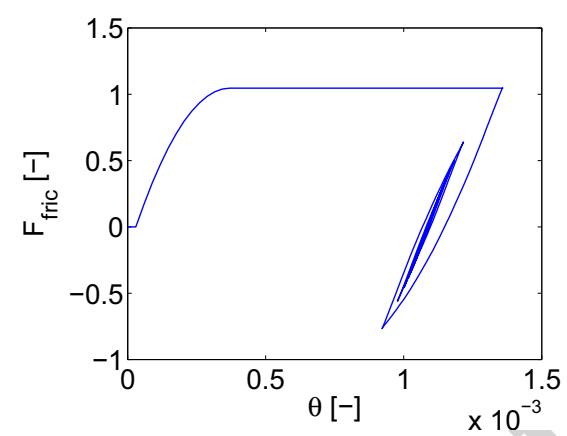

(b)

Figure 5: Free response of the system. (a) Phase portrait, (b) friction-force vs. angular displacement.

for lightly damped systems.

\subsection{Simulation results}

Figure 5(a) depicts the phase plot of the free response, with initial conditions $\dot{\theta_{0}}=0.3$ and $\theta_{0}=0$ and null initial traction field. In the nomenclature section, an additional table is added with the numerical values of the variables used in the simulations. The amplitude dependency of the damping is clearly visible. In Figure 5(b), the friction force is plotted as function of the ball rotation angle. During the oscillatory decay, the increasing stiffness of the hysteresis behavior is observed, as evidenced by the ever increasing slope of force-displacement curve.

When we apply a ramp input of the position $x_{t}$, that is, a step input of the velocity $\dot{x}_{t}$, we may expect damped oscillatory behavior of the rotational velocity $\dot{\theta}$ of the sphere, while the angular position of a point on the surface of the sphere will lag the reference signal. In Figure 6, which simulates this situation, the angular position and velocity are plotted together with their reference signal. One can clearly see the resonant behavior of the ball in the velocity signal. The resonance originates from the ball inertia and the tangential stiffness in the contact patch. 


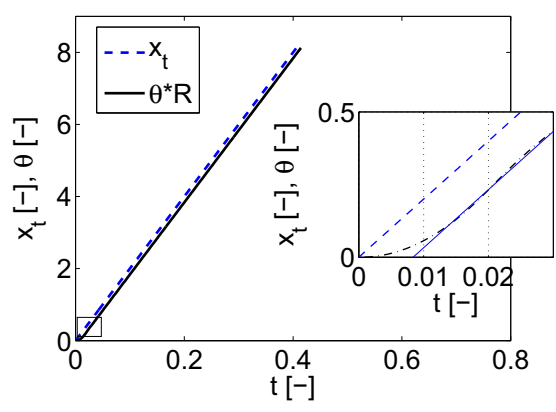

(a)

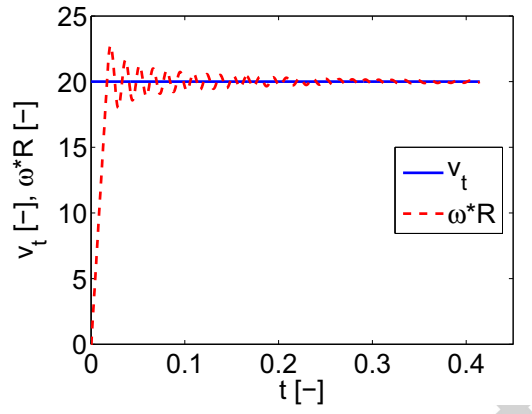

(b)

Figure 6: Ramp input to the position of the plate $x_{t}$. (a) the ball lags the plate, (b) damped oscillations of the ball.

To perform a systematic analysis of the nonlinear behavior, a stepped-sine excitation is applied to the system. That is, the upper plane motion $x_{t}$ is made sinusoidal, for a set of discrete frequencies. This experiment is carried out for different excitation amplitudes, in order to study the influence of the amplitude on the behavior. In Figure $7(\mathrm{a})$, the frequency response function $F_{\text {fric }} / x_{t}$ is plotted, which represents a measure of the complex stiffness of the ball-flat contact. For low frequencies, one can notice an increasing stiffness, up to a certain break frequency after which the stiffness becomes constant and a phase lag begins. For low frequencies, the motion of the plate is (almost) completely transmitted to the the ball, which can thus follow the imposed motion. For higher excitation frequencies, the velocity of the plate increases and as a result, the ball will no longer be able to follow the plane's motion, which results in a certain amount of slip between plane and sphere. The transition frequency from increasing stiffness to a constant amplitude complex stiffness is denoted by $f_{s}$. In Fig. $7(\mathrm{~b}), f_{s}$ is plotted as a function of the excitation amplitude. One can notice a decreasing exponential trend for $f_{s}$ as function of $A$.

A profound study of this behavior in function of the different model parameters can result in a better insight into the problem and a consequently better design to avoid early wear and failure of the system due to excessive slip in the contacts. Because the model parameters are reduced by normalizing w.r.t. the radius of 


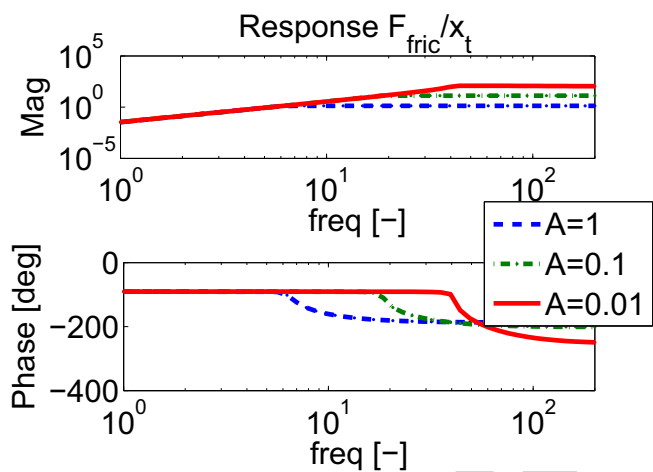

(a)

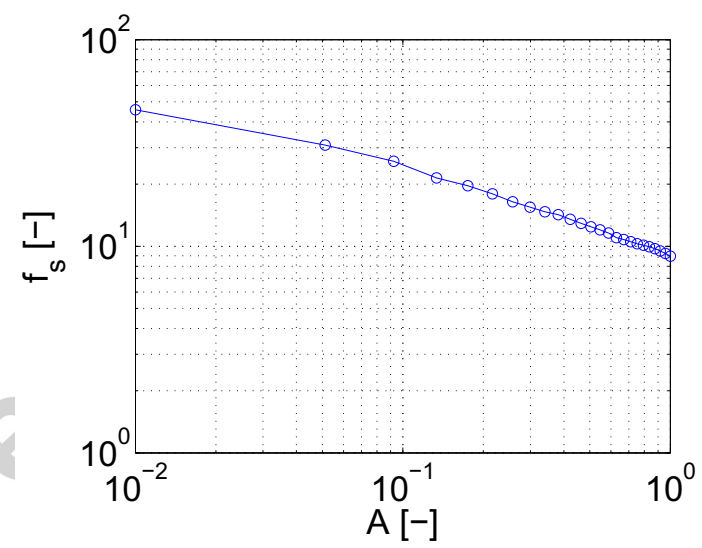

(b)

Figure 7: Frequency response function of the tangential contact stiffness. (a) complex stiffness $F_{x} / x_{t}$ as function of frequency for different excitation amplitudes $A$. (b) break frequency $f_{s}$ as function of excitation amplitude $A$. 
the contact patch $a$, the maximum normal pressure $p_{0}$, and the ball inertia $I$, these parameters fall out of the model equations, so that Eq. 1 becomes:

$$
1=\left(\frac{3 W^{*} R^{*}}{4 E_{h}^{*}}\right)^{1 / 3} \Leftrightarrow R^{*}=\frac{4 E_{h}^{*}}{3 W^{*}}
$$

In other words, $R^{*}$ increases if $E^{*}$ increases or $W^{*}$ decreases. In this way, $R^{*}$ contains all geometry, elasticity and load information. Since the ball inertia is also reduced by nondimensionalizing the model, we get

$$
I^{*}=1=R_{g}^{2} m^{*}=f\left(R^{*}\right) m^{*} \Rightarrow R^{*}=f\left(m^{*}\right)
$$

From Eq. 20, $R^{*}$ also holds the inertial or mass information. To study the influence of $R^{*}$, this parameter is varied between reasonable bounds. Owing to geometrical limitation, $a$ cannot be larger than about $R / 4$ without violating the assumptions of the Hertz theory.

The resonance frequency $f_{\text {res }}$ is defined as:

$$
f_{\text {res }}=\frac{1}{2 \pi} \sqrt{\frac{R^{* 2} K^{*}}{I^{*}}}
$$

where $K^{*}$ is the tangential stiffness in the contact patch.

\subsection{Dynamic behavior of a mass on a nonlinear element}

In this section, the dynamic behavior is discussed when non-vanishing inertia, a (dimensionless) mass $M$, is assigned to the plate. In this case, the input force $F_{x}$, needed to maintain a sinusoidal displacement $x_{t}$ (applied to the plate, as in the previous section), is function of the inertia of the plate. Figure 8 shows the absolute frequency response function of the simulated force/displacement for an indicated excitation amplitude $A=1$, for different masses of the plate. As can be seen, the shape of the transfer function changes drastically with changing plate mass. Obviously, the limit $M=0$ corresponds to Fig. $7(\mathrm{a})(A=1)$, while for $M \rightarrow \infty$, the mass-line limit is approached. 


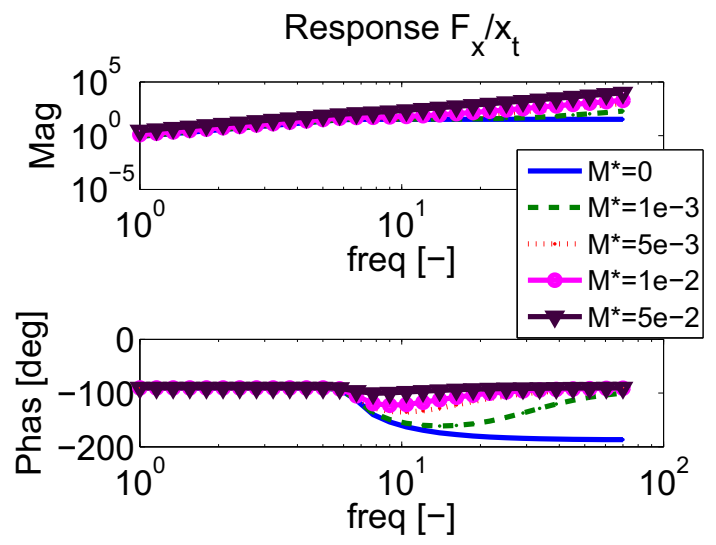

Figure 8: The frequency response function for different plate masses $\left(A^{*}=1\right)$.

\section{Case 2: A rolling sphere in between two planes}

\subsection{System description}

The system of a rolling sphere in between two flat plates is depicted in Fig. 9. This is a simplified model representing various kinds of rolling, such as that found in ball bearings, linear guideways, cam-follower systems, etc. The system is excited by applying a tangential displacement to the upper plate, while the motion of the lower plate is obstructed. The friction force in the upper contact is denoted by $F_{x 1}$, while the friction force in the lower contact is denoted by $F_{x 2}$. The creepage in each contact is given by Eq. 22 .

$$
\begin{aligned}
& \xi_{1}=\frac{\dot{x_{t}-(\dot{x}+R \dot{\theta})}}{\dot{x}_{t}} \\
& \xi_{2}=\frac{-(\dot{x}-R \dot{\theta})}{\dot{x}}
\end{aligned}
$$

\subsection{Simulation results}

When a velocity step is applied to the system, the (exponentially windowed) spectrum of $F_{x 1}$ shows two peaks, originating from the natural frequencies of the translation and rotation oscillations, respectively, given by

$$
\begin{aligned}
& \omega_{1}=\sqrt{\frac{K_{u p}^{*}+K_{l o}^{*}}{m^{*}}} \\
& \omega_{2}=\sqrt{\frac{R^{* 2}\left(K_{u p}^{*}+K_{l o}^{*}\right)}{I^{*}}},
\end{aligned}
$$




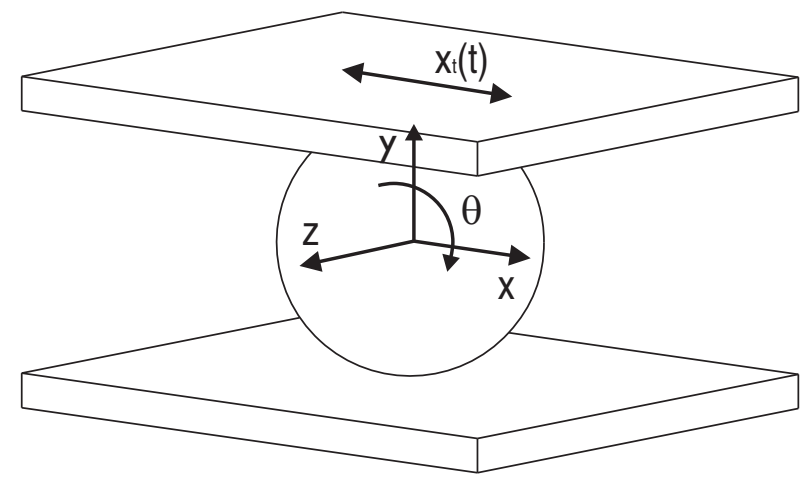

Figure 9: Rolling dynamics case 2:A 2-DOF system: rotation and translation.

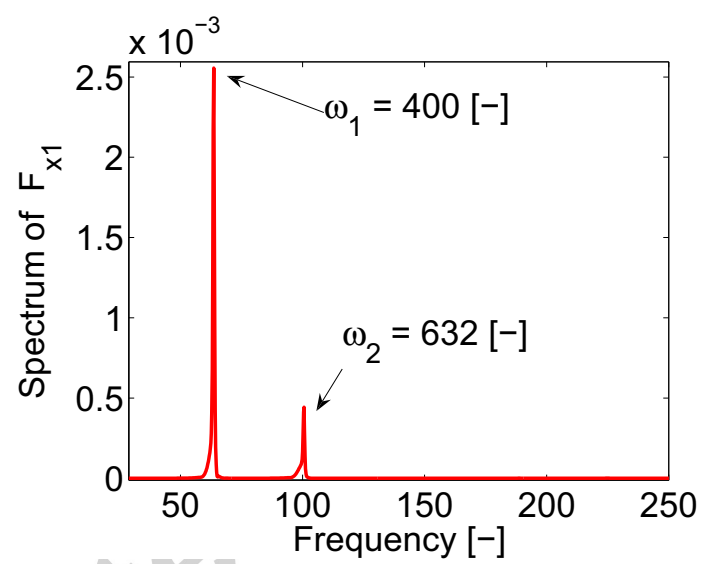

Figure 10: Spectrum of $F_{x 1}$. The two distinct peaks represent the translation and the rotation resonance frequencies.

where, $K_{u p}^{*}$ and $K_{l o}^{*}$ are the tangential contact stiffness in the upper and lower contact patches, respectively. These stiffnesses can be calculated from the tractive behaviour in each contact. Since for a solid sphere $I^{*}=2 / 5 m^{*} R^{* 2}$ and under the assumption that $K_{u p}^{*}$ equals $K_{l o}^{*}$, one can derive that $\omega_{2}=\sqrt{5 / 2} \omega_{1}$. This is confirmed by the simulation results of Fig. 10 .

The response to a velocity step input, is given in Fig. 11. From these plots, one can see the different frequencies of the rotational and the translational motion of the sphere. $v_{1}$ and $v_{2}$ are the velocities of particles on the surface of the sphere, in the upper and lower contact, respectively. Since $v_{1}=\dot{x}+\dot{\theta} R$ and 


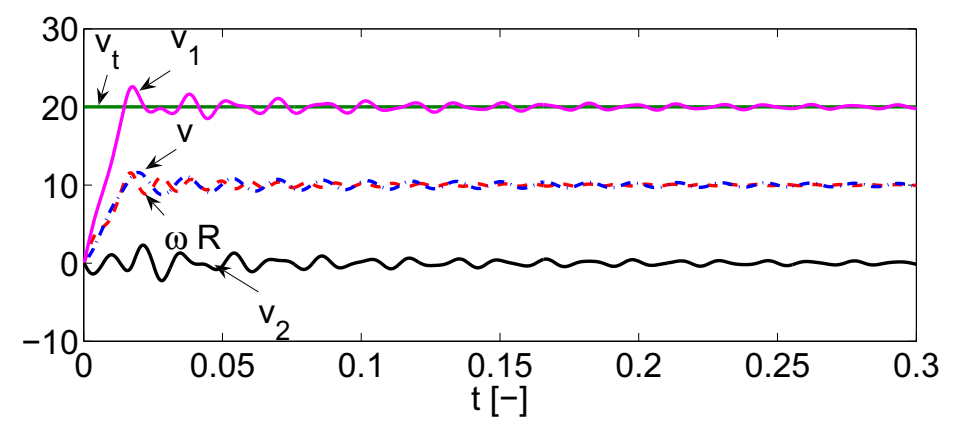

Figure 11: Rolling dynamics case 2: A velocity step response.

$v_{2}=\dot{x}-\dot{\theta} R$, these particle velocities contain frequency information from both modes. $\left(v_{1}-\dot{x}_{t}\right)$ and $v_{2}$ denote the slipping velocities in both contacts and account for the energy dissipation and consequently for damping and wear in both contacts.

In Figure 12, three sinusoidal excitations $x_{t}=A \sin \left(2 \pi f_{t} t\right)$ with different excitation frequencies are compared. The initial velocities $\dot{\theta}(0)$ and $\dot{x}(0)$ are set equal to $2 \pi f_{t} A /(2 R)$ and $2 \pi f_{t} A / 2$ respectively, to avoid transient effects at the start of the simulation. For the lowest excitation frequency $f_{t 1}=1$, Fig. 12(a), there is almost no sliding in the contacts. When increasing the frequency to $f_{t 2}=6.5$, the sliding increases, Fig. 12(c). For yet higher excitation frequency $f_{t 3}=10$, Fig. $12(\mathrm{e})$, high sliding speeds occur in the contacts, while the motion of the sphere, both for rotation and translation, is no longer sinusoidal, but approximates a rounded triangular signal. The friction forces in both contacts are visualized for these three cases in the right column of Fig. 12(b,d,f).

When applying a stepped-sine excitation to the system, identical results are obtained as for the system with one degree-of-freedom of section 3. We can therefore conclude that the translating motion does not influence the stiffness properties of the upper contact. 


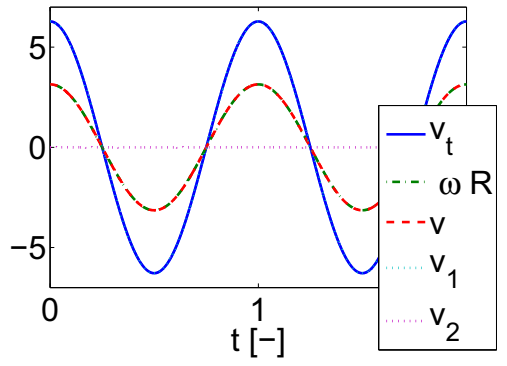

(a)

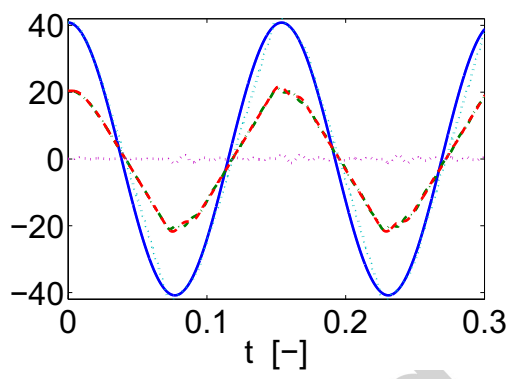

(c)

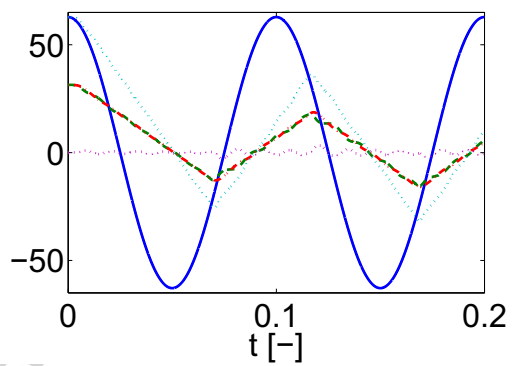

(e)

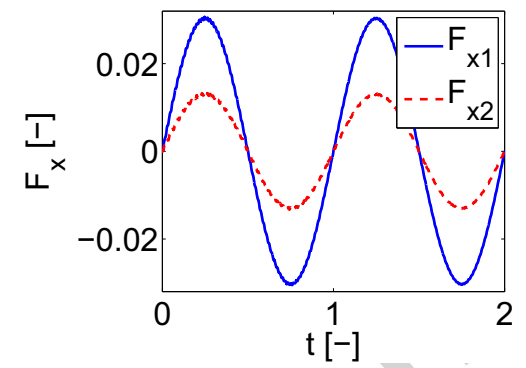

(b)

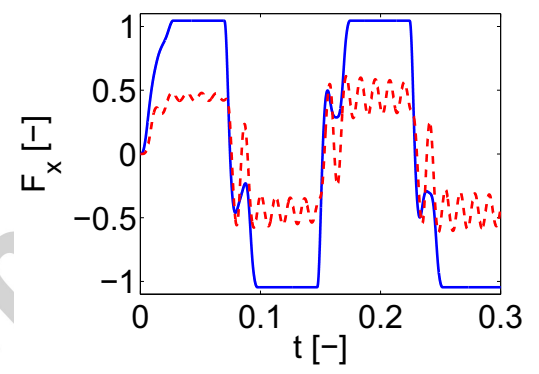

(d)

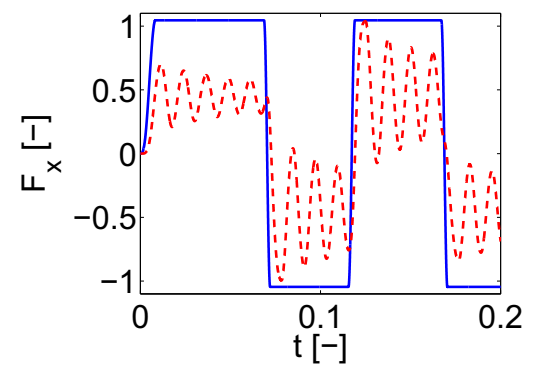

(f)

Figure 12: Sinusoidal excitation with different frequencies $f_{t}$. (a) motion response for $f_{t}=1$, (b) friction force in the contacts for $f_{t}=1$, (c) motion response for $f_{t}=6.5$, (d) friction force in the contacts for $f_{t}=6.5$, (e) motion response for $f_{t}=10$, (f) friction force in the contacts for $f_{t}=10$. 


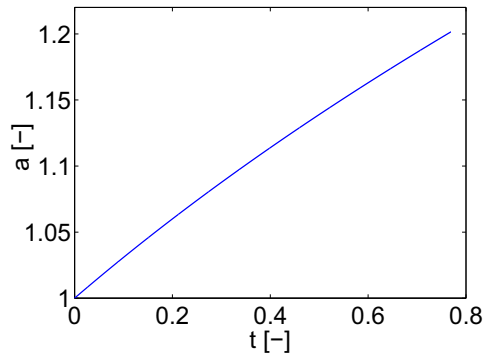

Figure 13: Rolling in between two plates with sinusoidal excitation of the top plate and increasing normal load: increasing dimensionless contact patch radius as function of time corresponding to the linearly increasing dimensionless external load.

In a significant part of the applications, the rolling element is subjected to a varying normal load, implying a variable contact patch. An obvious example is the loaded deep groove ball bearing, provided with a static radial load. Here, the load for each ball in the bearing alters with its changing radial position. Fig. 13 shows the evolution of the contact patch radius as function of time. In Fig. 14, the frictional forces are plotted as function of time, when the externally applied force $W^{*}$ changes progressively with time, from 2 up to 3.6. From Fig. 14, one can notice the beneficial effect of the increasing normal load on the damping of high frequency components.

\section{Case 3: A rolling sphere in between two V-grooved tracks}

\subsection{System description}

Figure 15 shows the configuration of a sphere rolling in between two Vgrooves. To the upper $\mathrm{V}$-groove, a displacement $x_{t}(t)$ is applied, while the lower V-groove stands still. From the right panel of Fig. 15, one can notice that the rolling motion of the ball $\omega$ can be resolved in the contact patches as a spin motion $\omega_{s}$ combined with a pure rolling motion $\omega_{r}$. In a kinematic analysis, 


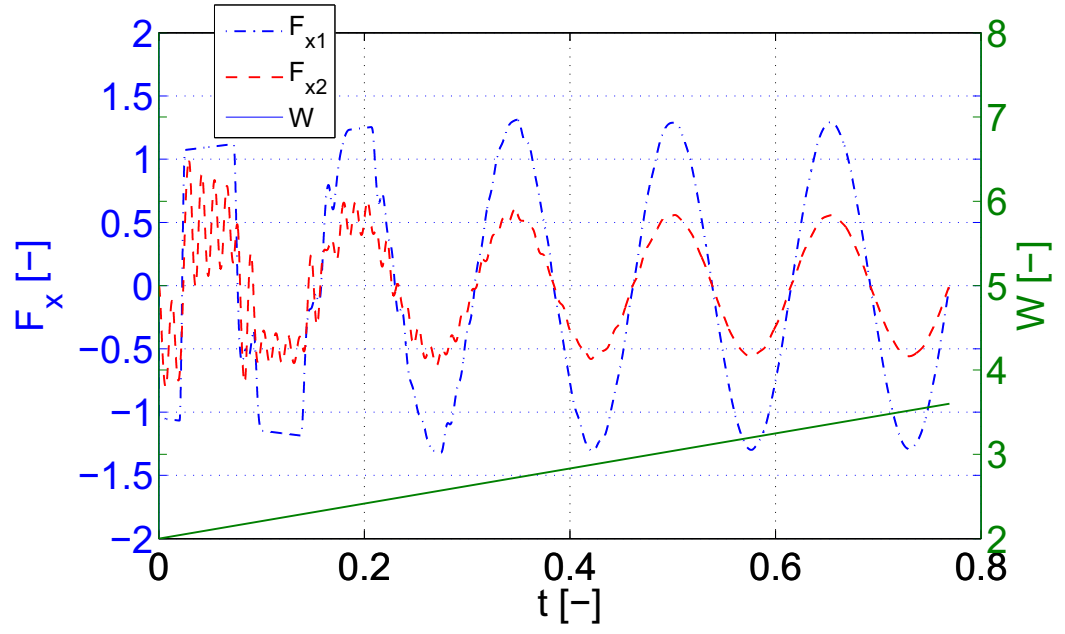

Figure 14: Rolling in between two plates with sinusoidal excitation of the top plate and increasing normal load: evolution of the friction force in the upper $\left(F_{x 1}\right)$ and lower $\left(F_{x 2}\right)$ contact.
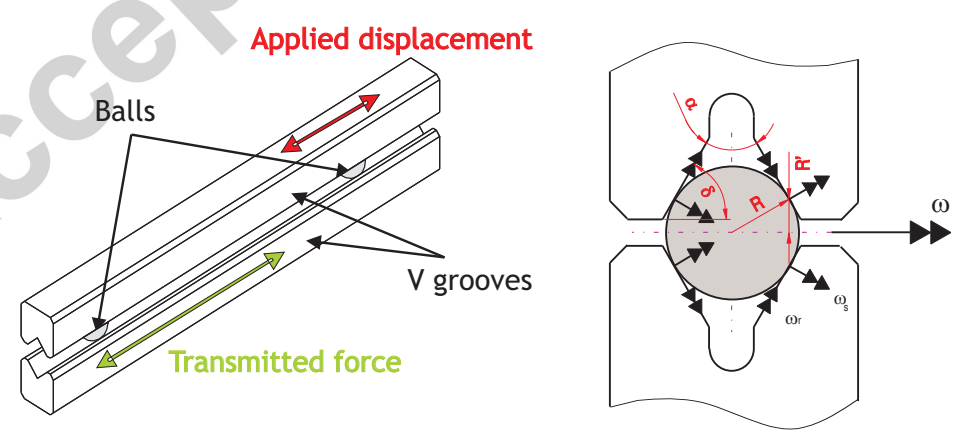

Figure 15: Rolling dynamics case 3: V-groove rolling (reproduced with permission from [24]). 


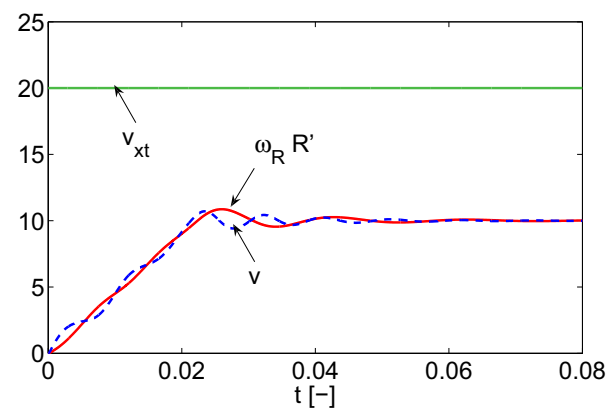

Figure 16: Step response to a velocity step.

that is, where inertial effects are neglected, the spin motion of the ball is the main source for the frictional losses in the contact. For a low-frequency excitation of the upper V-groove, the spin motion is determined by the geometry of the system, i.e. the ball radius $R$, and the opening angle of the $\mathrm{V}$-groove, $\alpha$. Analysis of rolling in V-groove can be found in [24], where experimental evidence, pertaining to low frequency motion, is weighted against the theoretical model briefly presented in section 2. For higher dynamical excitation, the inertia of the sphere will cause an additional creepage in the contact patches and consequently extra losses.

The spin component is given by [24]:

$$
\phi=\frac{\omega_{s}}{v_{t}}=\frac{\omega \cos \left(\frac{\alpha}{2}\right)}{v_{t}}
$$

\subsection{Simulation results}

The motion response to a step input of the velocity $\dot{x_{t}}$ is presented in Fig. 16, with groove angle $\alpha=\frac{\pi}{3}$. The transient is damped out much faster than in the case of systems 1 and 2 (cf. sections 3 and 4) because of the extra, permanent damping due to the spinning motion in the contact patches. The spin component, and consequently the extra damping, decrease with increasing groove angle $\alpha$. Due to the geometry of the system, the natural frequencies of the translation and rotation oscillations are given by

$$
\begin{aligned}
& \omega_{1}=\sqrt{\frac{K_{u p}^{*}+K_{l o}^{*}}{m^{*}}} \\
& \omega_{2}=\sqrt{\frac{R^{\prime * 2}\left(K_{u p}^{*}+K_{l o}^{*}\right)}{I^{*}}}=\sqrt{\frac{R^{* 2} \sin ^{2}\left(\frac{\alpha}{2}\right)\left(K_{u p}^{*}+K_{l o}^{*}\right)}{I^{*}}},
\end{aligned}
$$


with $K_{l o}^{*}$ and $K_{u p}^{*}$ the tangential contact stiffness in the lower and upper contact respectively, which results in $\omega_{2}=\sqrt{5 / 2} \sin (\alpha / 2) \omega_{1}$, assuming that $K_{u p}^{*}$ equals $K_{l o}^{*}$. Thus, when $\sqrt{2.5} \sin (\alpha / 2)>1$, the resonance frequency of the rotational mode $\omega_{2}$ is higher than the translational model $\omega_{1}$. The flipping point of the modes, that is, the point at which the two frequencies become equal, lies at $\alpha=78.46^{\circ}$.

The frequency-response characterization of an externally excited nonlinear dynamic system can only be carried out in an approximating way, since analytical solutions are not available [29]. One of the methods is the describing function method. Here, the nonlinear element is replaced by an element that gives the fundamental component in Fourier terms, of the output of that non-linear element for a sinusoidal input. In [22], this type of analysis is carried out to obtain three characterizing parameters of the hysteresis loop. The equivalent stiffness $k_{e}$ and the equivalent damping, $c_{e}$, which give rise to an excitation dependent equivalent damping ratio $\zeta_{r}$. The equations obtained in [22] are

$$
\begin{array}{r}
k_{e} \approx \frac{F_{m}}{A_{m}} \\
c_{e} f=\frac{\text { loop area }}{\pi A_{m}^{2}} \\
\zeta_{r}=\frac{\text { loop area }}{2 \pi A_{m} F_{m}}
\end{array}
$$

Since these three parameters comprise the hysteresis characteristic behavior, a study of these shows the dependence of the characteristics on the influence parameters. From the previous section, it is clear that the dimensionless ball radius $R^{*}$ comprises load information, geometrical and material properties. The local coefficient of friction $\mu$, forms an additional influence parameter which, together with the $\mathrm{V}$-groove angle of the system $\alpha$, and the ball radius, influences the spin creepage $\phi^{*}$ in the contact patches. The influence of these parameters on the equivalent stiffness and damping ratio of the hysteresis are depicted in Fig. 17. The equivalent stiffness $k_{e}$ and the equivalent damping $c_{e} f$ decrease with increasing ball radius $R^{*}$ and increasing groove angle $\alpha$, and increase with increasing coefficient of friction $\mu$. The damping ratio decreases with increasing 
ball radius, increasing coefficient of friction and increasing groove angle.

For a sinusoidal excitation $x_{t}$, at low frequencies, the system behaves quasi similar to the kinematic model described in [24], since the creepage in the contact originates almost only from the spin due to rolling in a V-groove. When the excitation frequency is increased, however, extra creepage will occur in the contacts due to the inertial effect of the ball. For very high frequencies, the latter effect will dominate. In Figure 18, the hysteresis curve is plotted for different excitation frequencies for a constant excitation amplitude. One can clearly see the increasing stiffness of the hysteresis loop with increasing excitation frequency $f_{t}$. However, as the frequency increases further, the hysteresis behavior becomes distorted and shows chaotic behavior, as shown in Fig. 19, where the force-displacement plot is depicted for an amplitude $A$ of 1 and a frequency $f_{t}$ of 5. The combination of sliding due to the inertial effect of the ball, together with the spinning motion of the ball, results in an additional oscillatory behavior. Finally, in Figure 20, the equivalent stiffness and equivalent damping ratio are plotted as function of the excitation frequency and the excitation amplitude. The equivalent stiffness $k_{e}$ increases with increasing excitation frequency $f_{t}$, while the latter has no significant effect on the equivalent damping $c_{e} f$. Thus, the damping ratio $\zeta_{r}$ decreases with increasing excitation frequency. However, the results shown in Fig. 20 are no longer valid for high excitation frequency and large amplitudes, since regular hysteretic behavior is absent in that region of operation (see Fig.19).

\section{Conclusions}

In this paper, the simulation of transient rolling friction is reported. The interplay between friction and the other system dynamics is investigated.

Firstly, a brief introduction is given to to rolling friction model, which enables 


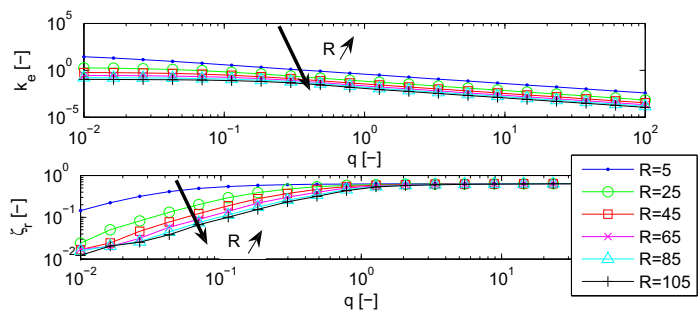

(a)

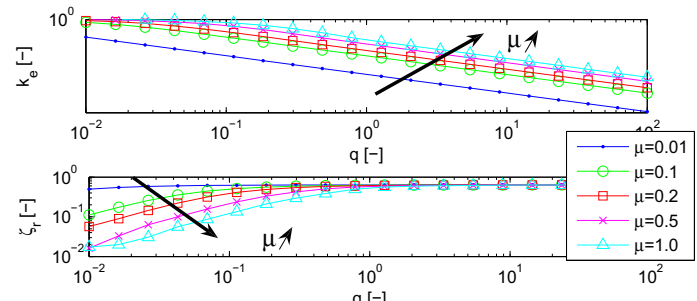

(b)

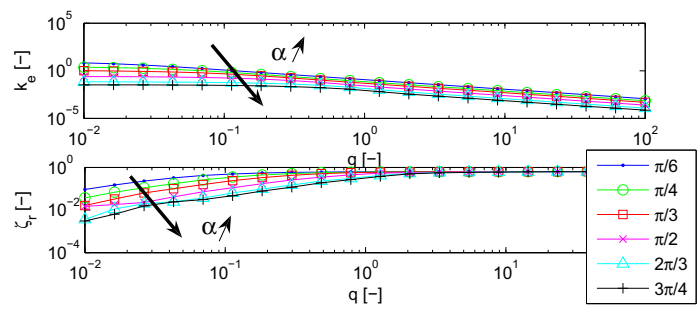

(c)

Figure 17: Equivalent stiffness $k_{e}$ and equivalent damping ratio $\zeta_{r}$ as function of excitation amplitude for (a) different dimensionless ball radii, (b) different coefficients of friction and (c) different $\mathrm{V}$-groove opening angles. 


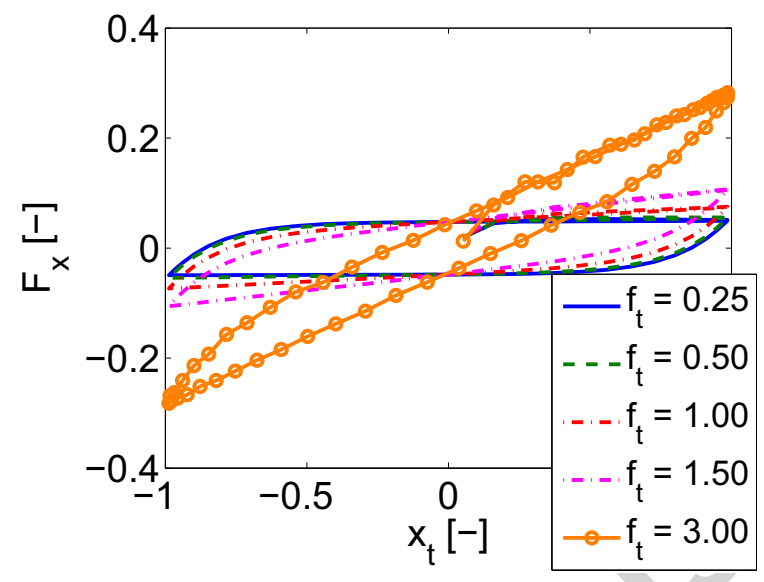

Figure 18: The hysteresis loop for different excitation frequencies $f_{t}\left(\alpha=\frac{\pi}{3}\right)$.

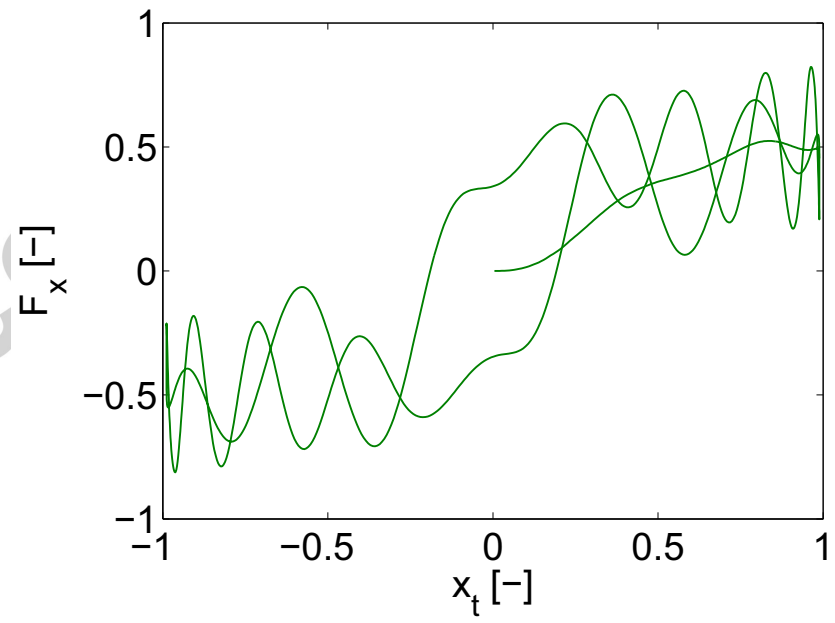

Figure 19: The displacement-force plot for $f_{t}=5$. 

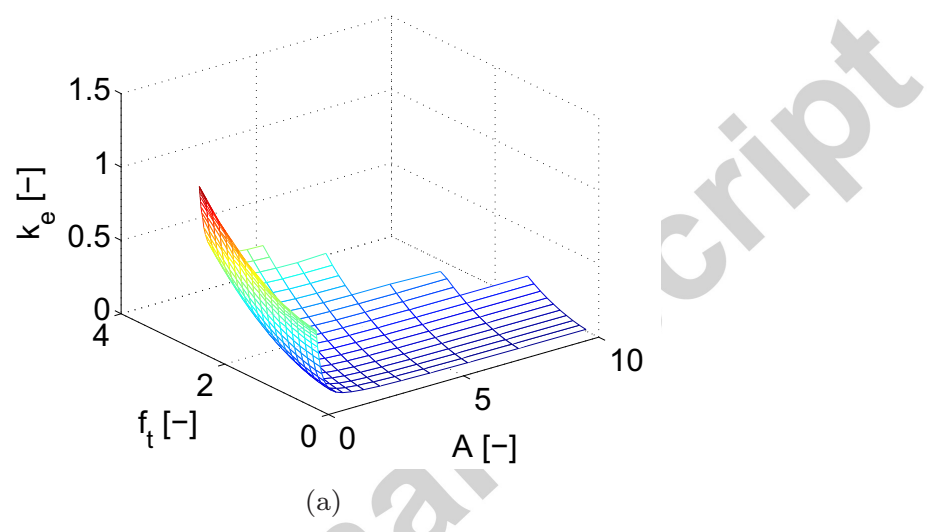

(a) 20

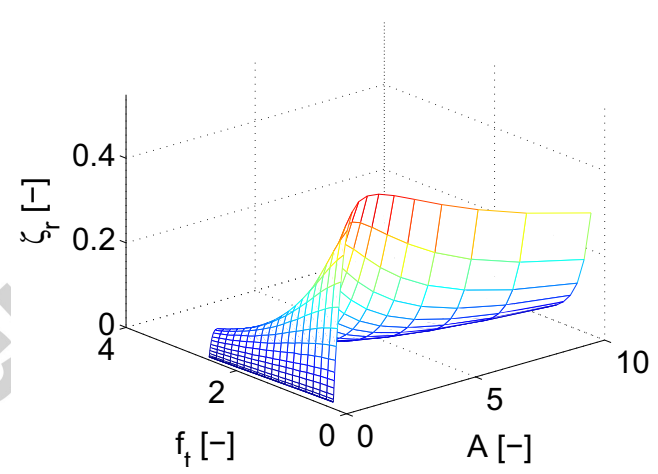

(b)

Figure 20: Equivalent stiffness $k_{e}$ and equivalent damping ratio $\zeta_{r}$ as function of excitation amplitude $A$, and excitation frequency $f_{t}$. 
one to simulate the transient traction stresses in the contact of a rolling body and upon a counter surface. Thereafter, that model is used in the dynamic simulation of systems comprising rolling elements. The basic characteristics of such systems, such as the traction forces in the contact and the resulting complex stiffness are examined. It turns out that systems comprising rolling elements can manifest complex dynamics that depends on the amplitude and frequency of excitation.

For the case of a ball rolling on a massless plate, an increasing complex stiffness as a function of excitation frequency is observed, up to a certain break frequency. For higher frequencies, the amplitude of the complex stiffness becomes constant. The influence of plate inertia, was also investigated in the time and the frequency domains.

Finally, owing to its relevance in industrial applications, a system of a ball rolling between two V-grooved tracks is investigated, where a combination of kinematic creepage and dynamic creepage occurs. The study of these dynamic effects in frictional contacts, can significantly help improve the position accuracy of systems comprising these elements. Furthermore, a profound knowledge of the frictional dissipation in the rolling contacts, such as given in this paper, permits a better estimation of lifetime and performance degradation due to fatigue and wear. 


\section{Appendix A: Nomenclature}
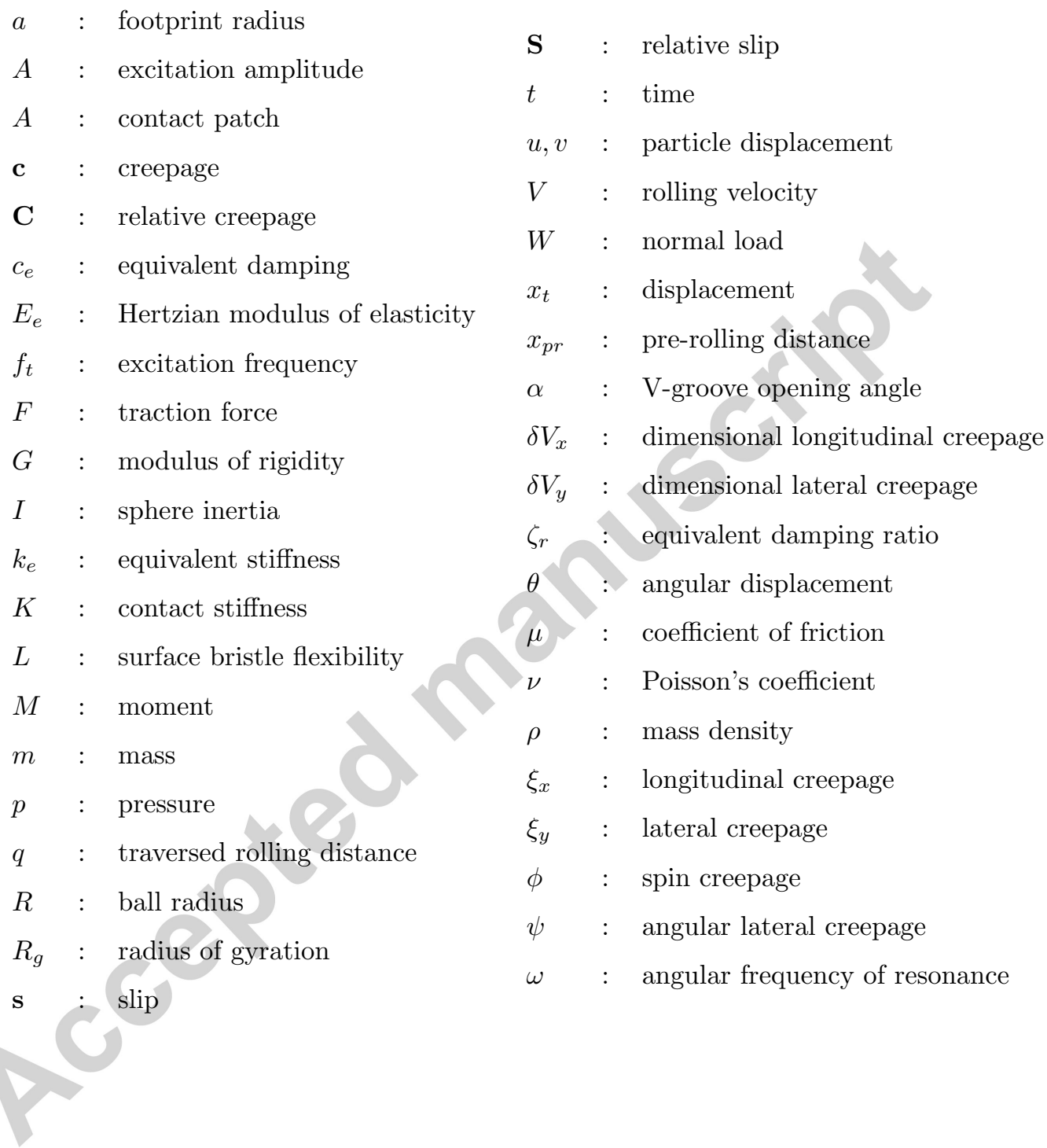


\begin{tabular}{|l|l|l|l|}
\hline dimensional variable & value & dimensionless variable & value \\
\hline$a$ & $1.4812 \mathrm{e}-4 \mathrm{~m}$ & $a^{*}$ & $1[-]$ \\
$E$ & $1.1538 \mathrm{e} 11 \mathrm{~Pa}$ & $E^{*}$ & $53.0227[-]$ \\
$I$ & $4.1103 \mathrm{e}-8 \mathrm{kgm}^{2}$ & $I^{*}$ & $1[-]$ \\
$m$ & $0.0041 \mathrm{~kg}$ & $m^{*}$ & $0.0022[-]$ \\
$p_{0}$ & $2.1761 \mathrm{e} 9 \mathrm{~Pa}$ & $p_{0}^{*}$ & $1[-]$ \\
$R$ & $0.005 \mathrm{~m}$ & $R^{*}$ & $33.7553[-]$ \\
$W$ & $100 \mathrm{~N}$ & $W^{*}$ & $2.0944[-]$ \\
$\mu$ & $0.5[-]$ & $\mu^{*}$ & $0.5[-]$ \\
$\nu$ & $0.3[-]$ & $\nu^{*}$ & $0.3[-]$ \\
\hline
\end{tabular}

\section{Appendix B: The flexibility parameter}

With $G$ being the equivalent modulus of rigidity of the sphere, $L_{1}, L_{2}$ and $L_{3}$ are obtained from [30] as:

$$
\begin{aligned}
L_{1} & =\frac{8 a}{3 G C_{11}} \text { with } C_{11}=4.34 \\
L_{2} & =\frac{8 a}{3 G C_{22}} \text { with } C_{22}=3.73 \\
L_{3} & =\frac{\pi a^{2}}{4 G a C_{23}} \text { with } C_{23}=1.50
\end{aligned}
$$

\section{Acknowledgements}

This research is supported by the Fund for Scientific Research - Flanders (F.W.O.) under Grant FWO4283. The scientific responsibility is assumed by its authors.

\section{References}

[1] S Futami, A Furutani, and S Yoshida. Nanometer positioning and its micro-dynamics. Nanotechnology, 1(1):31-37, 1990.

[2] B. A. Awabdy, W.-C. Shih, and D. M. Auslander. Nanometer Positioning of a Linear Motion Stage Under Static Loads. IEEE/ASME Transactions of Mechatronics, 3(2):113119,1998

[3] S. Mekid. High precision linear slide. Part I: design and construction. International Journal of Machine Tools and Manufacture, 40(7):1039-1050, 2000. 
[4] Y.-S. Yi, Y. Y. Kim, J. S. Choi, J. Yoo, D. J. Lee, S. W. Lee, and S. J. Lee. Dynamic analysis of a linear motion guide having rolling elements for precision positioning devices. Journal of Mechanical Science and Technology, 22:50-60, 2008.

[5] T. A. Harris. An Analytical Method to Predict Skidding in Thrust-Loaded, AlngularContact Ball Bearings. ASME Journal of Lubrication Technology, 93(1):17-24, 1971.

[6] T. A. Harris. Ball Motion in Thrust-Loaded, Angular Contact Bearings With Coulomb Friction. ASME Jounal of Lubrication Technology, 93(1):32-38, 1971.

[7] P. Szuminski. Determination of the Dynamic Angle of the Bearings Rolling Element Location and of Its Influence on the Loads and Rolling Friction. Journal of Robotic Systems, 17(12):671-687, 2000.

[8] Neng Tung Liao and Jen Fin Lin. A New Method for the Analysis of Deformation and Load in a Ball Bearing With Variable Contact Angle. Journal of Mechanical Design, 123(2):304-312, 2001.

[9] Neng Tung Liao and Jen Fin Lin. Ball bearing skidding under radial and axial loads. Mechanism and Machine Theory, 37(1):91 - 113, 2002.

[10] Neng Tung Liao and Jen Fin Lin. An Analysis of Misaligned Single-Row Angular-Contact Ball Bearing. Journal of Mechanical Design, 126(2):370-374, 2004.

[11] P. Szuminski. Determination of the stiffness of rolling kinematic pairs of manipulators. Mechanism and Machine Theory, 42:1082-1102, 2007.

[12] G. Heinrich and M. Klüppel. Rubber friction, tread deformation and tire traction. Wear, 265:1052-1060, 2008.

[13] J. H. Schutte, Y. H. Wijnant, and A. de Boer. A Contact Solver Suitable for Finite Elements. In P. Sas and B. Bergen, editors, Proceedings of the Internation Conference on Noise and Vibration Engineering ISMA2008, 2008.

[14] V. K. Garg and R. V. Dukkipati. Dynamics of Railway Vehicle Systems. Academic Press, Canada, 1984.

[15] A. Ekberg. Rolling contact fatigue of railway wheels - a parametric study. Wear, 211:280$288,1997$.

[16] R. Nilsson. On wear in rolling/sliding contacts. PhD thesis, KTH Machine design, 2005.

[17] J. B. Nielsen. New Developments in the Theory of Wheel/Rail Contact Mechanics. PhD thesis, Technical University of Denmark, Lyngby, Denmark, 1998.

[18] T. Telliskivi. Simulation of wear in a rolling-sliding contact by a semi-Winkler model and the Archard's wear law. Wear, 256:817-831, 2004.

[19] E. Cataldi-Spinola, C. Glocker, R. Stefanelli, and M. Götsch. Influence of the Wheel Diameter on the Curve Squealing of Railway Vehicles. In Euronoise 2003, 2003.

[20] J. F. Brunel, P. Dufrénoy, M. Naït, J. L. Muñoz, and F. Demilly. Transient model for curve squeal noise. Journal of Sound and Vibration, 293:758-765, 2006.

[21] S. S. Hsu, Z. Huang, S. D. Iwnicki, D. J. Thompson, C. J. C. Jones, G. Xie, and P. D. Allen. Experimental and theoretical investigation or railway wheel squeal. Proc Instn Mech Engrs : Part F: J. Rail and Rapid Transit, 221:59-73, 2007.

[22] F. Al-Bender and W. Symens. Characterization of frictional hysteresis in ball-bearing guideways. Wear, 258:1630-1642, 2005. 
[23] F. Al-Bender and K. De Moerlooze. A model of the transient behavior of tractive rolling contacts. Advances in Tribology, (vol. 2008):17, 2008.

[24] K. De Moerlooze and F. Al-Bender. Experimental investigation into the tractive prerolling behavior of balls in V-grooved tracks. Advances in Tribology, (vol. 2008):10, 2008 .

[25] P. K. Gupta. Advanced Dynamics of Rolling Element Bearings. Springer, Berlin, 1984.

[26] R. Abascal. 2D transient dynamic friction contact problems. I. Numerical analysis. Engineering Analysis with Boundary Elements, 16:227-233, 1995.

[27] C. Spiegelberg, S. Björklund, and S. Andersson. Simulation of transient friction of a cylinder between two planes. Wear, 254:1170-1179, 2003.

[28] N. M. Newmark. A Method of Computation for Structural Dynamics. ASCE Journal of the Engineering Mechanics Division, 85:67-94, 1959.

[29] F. Al-Bender, W. Symens, J. Swevers, and H. Van Brussel. Theoretical analysis of the dynamic behavior of hysteresis elements in mechanical systems. International Journal of Non-Linear Mechanics, 39:1721-1735, 2004.

[30] J. J. Kalker. Three-dimensional elastic bodies in rolling contact. Kluwer, Dordrecht, 1990. 